\title{
TOBACCO MOSAIC VIRUS BIOTEMPLATED ELECTROCHEMICAL BIOSENSOR
}

\author{
Hadar Ben-Yoav ${ }^{l^{*}}$, Adam D. Brown ${ }^{2}$,Ekaterina Pomerantseval ${ }^{1}$, Deanna L. Kelly, James N. Culver ${ }^{2}$,
} and Reza Ghodssi $i^{*}$

${ }^{1}$ MEMS Sensors and Actuators Laboratory, Department of Electrical and Computer Engineering, Institute for

Systems Research,

\author{
${ }^{2}$ Institute for Bioscience and Biotechnology Research, University of Maryland, College Park, Maryland, USA
}

${ }^{3}$ Maryland Psychiatric Research Center, University of Maryland School of Medicine, Baltimore, Maryland, USA

\section{ABSTRACT}

This work demonstrates the first utilization of virus molecules as nano-scale biotemplates assembled on an electrochemical biosensor, allowing for an 8-times increased signal and an improved biosensing performance of 9.5 -fold. The versatile and inexpensive biological Tobacco mosaic virus was integrated as a high aspect ratio, low footprint, low-cost, easy to genetically functionalize, nanostructured three-dimensional scaffold for the synthesis of novel multifunctional electrodes. The biotemplated scaffold allows for an increased surface area resulting in higher electrochemical currents, better signal-to-noise ratio and improved sensitivity when incorporated into miniaturized biosensors.

\section{INTRODUCTION}

\section{Electrochemical Biosensors}

Electrochemical biosensors are based on a bioelectrochemical interaction process, where electrochemical species are consumed or generated. Most electrochemical measurements detect oxidation/reduction of the product generated by biological conversion of the analyte undergoing redox reactions on the sensor's surface and solely respond to electro-active species. Electrochemical measurements are classified according to the variable being measured: amperometry, potentiometry and conductometry $[1,2]$.

In recent decades, nanostructured materials have received much attention due to unique properties they offer as robust platforms for electronic and optical signal transduction. One of its major contributions is the design of a new generation of miniaturized biosensing devices. Furthermore, biofunctional nanoparticles can produce a synergistic effect between catalytic activity, conductivity, and biocompatibility to accelerate the signal transduction, leading to the quick development of stable, specific, selective and sensitive biosensors in different fields. An important point besides the biosensing applicability of nanomaterials is the use of manipulation techniques for their integration in fabrication techniques [3]. By integrating with electrochemical sensor fabrication, these materials provide interesting properties such as increased surface area. The augmented surface area leads to higher signal-to-noise ratio, increased sensitivity and dynamic range, short distances for mass transport and charge transfer as well as their ability to create complex nano-bio-architectures that allow volume change and unique selective biological functionality. Nanomaterials allow the development of new architectures applied in electrochemical sensing and biosensing devices [4, 5]. For example, $\mathrm{Ye}$ and colleagues presented a multi-walled carbon nanotube (MWNT)-based electrochemical biosensor for glucose detection [5]. The higher surface area of the well-aligned MWNT generated higher electrochemical currents that were correlated to a highly sensitive sensor.

\section{Tobacco mosaic virus}

Biological nanomaterials provide a versatile and cost effective solution for the fabrication of high surface area nanoarchitectures. One category of these biological nanostructures is plant and bacteria virus particles consisting of macromolecular assemblies of nucleic acid packaged by many copies of coat proteins. These molecules display some unique advantages as they show exceptional stability in a wide range of temperatures and $\mathrm{pH}$ values, in addition to their surface-exposed functional groups, selfassembly and tunability [6].

Among the available plant viruses [7], Tobacco mosaic virus (TMV) is the most extensively studied filamentous plant structure for nanoscale applications. The TMV virion is a rigid rod consisting of about 2,130 identical coat protein subunits stacked in a helix around a single strand of plus sense RNA, forming a $4 \mathrm{~nm}$ diameter channel through the $300 \mathrm{~nm}$ long virion axis. Properties of the TMV system that make it particularly useful as a selfassembling macromolecular template for nanomaterials include: 1) its known three-dimensional structure [8]; 2) a wealth of biophysical information on its self-assembly characteristics [9]; 3) the availability of creating novel virus structures and surfaces via established molecular techniques [10]; 4) a wide range of existing coat protein variants with diverse assembly properties [11], and 5) the ability to easily purify large quantities of virus and coat protein from infected plants. TMV-structured metal and metal-oxide nanowires have been synthesized using several techniques [12, 13], transport properties have been studied [14], and potential applications in nano-scale devices have been investigated through proof-of-concept demonstrations [15].

Previous work with engineered mutations of the TMV has resulted in enhanced particle coatings and templates that can be readily integrated into microfabricated devices and has established a novel patterning process. Efficient templates for metallic coatings have been achieved through the introduction of one (TMV-1cys) or two (TMV-2cys) cysteine residues within the coat protein open reading frame. Cysteines are amino acids with thiol groups that show enhanced metal binding properties based on strong, covalent-like interactions. One and two-step electroless plating methods have been used for the fabrication of TMV-2cysbased wires coated with gold, silver and palladium clusters that show more uniform coating compared to the wild-type virus [16]. Additionally, the rod-shaped viruses can be directionally attached to various surfaces and coated to create high aspect ratio nickel, cobalt and platinum materials (TMV-1cys). Alternative pathways were explored for patterning the viral molecules in microfabricated electrodes as well as controlled environments, utilizing nucleic acid hybridization [17]. Recent work in our team has focused upon the development of novel inorganic structures using the TMV and their application in energy storage devices. A simple and versatile approach for the selective patterning of both metal-coated and uncoated TMV using lift-off processing has been developed [18]. The high aspect ratio of the coated TMV in addition to its robustness was utilized in the development of high surface area nickel-zinc [19] and Li-ion microbatteries [20, 21]. By the integration of high aspect ratio biotemplated TMV scaffold in electrode fabrication process, high surface area electrochemical sensors can be realized improving the overall biosensing performance. 


\section{Mental Health Applications}

The medical management of mental health care is one of the most debilitating and costly of all disorders. A major unmet need is the ability to have an objective, real time analysis of the disorder at the point-of-care. Schizophrenia is a common mental health disorder, has high social and economic impact, and manifests early - mostly during adolescence and early adulthood. It is frequently preceded by premorbid pattern of rather unspecific but nevertheless handicapping symptoms that may be present for several years before clinical manifestation. Schizophrenia is a complex disorder involving difficulties with reality distortion, cognitive impairments, and work and social dysfunction. Often, it is also associated with symptoms of moodiness and anxiety. Once diagnosed, patients need to remain on antipsychotic medications lifelong. One of the biggest challenges of treatment is that many people discontinue their treatment which can lead to relapse and rehospitalizations, having a major impact on patient and society healthcare costs [22, 23]. Finding a method for continually monitoring patient status would allow for psychiatrists to more effectively treat these mental disorders.

Over the past few years, genetic analysis has found a strong relation between the gene encoding for Neuregulin-1 (NRG1) and schizophrenia [24]. Studies have obtained supporting evidence for NRG1 as a candidate gene for the disorder [25]. Moreover, the identification of NRG1 receptor ErbB4 as an additional candidate risk gene for schizophrenia strongly suggests that this signaling pathway participates in the pathophysiology of the disorder [26]. By monitoring NRG1 concentration during various stages of schizophrenia, mental health care management can be studied and improved.

Microfluidic lab-on-a-chip (LOC) microsystems provide numerous advantages in clinical diagnostics, environmental monitoring and biomedical research fields. These translational technologies hold potential to improve upon the resolution, regulation, sensitivity, flexibility, and cost-savings over more traditional approaches, bringing bench top methods into the pointof-care. By the integration of MEMS sensing devices with mental health analysis, pre-clinical assessments and the overall chance of success of personalized medical care at the point-of-care can be significantly improved.

\section{RESULTS}

One of the challenges with miniaturized biosensing devices is the high background signal and low signal-to-noise ratio that decrease the performance of biosensors. By increasing the signal, electrochemical biosensors can improve their bio-detection efficiency resulting in higher sensitivity. Here, we used gold planar square electrodes $(20 \mathrm{~nm} \mathrm{Cr} / 180 \mathrm{~nm} \mathrm{Au}$, surface area $=0.49$ $\mathrm{cm}^{2}$ ) that were patterned onto a silicon dioxide substrate via DC sputtering and were realized using both wet etching and lift-off techniques. Virus modification of the electrode surface was performed to enhance the total surface area. Fabrication was carried out by submerging the electrodes in a solution of rodshaped $300 \times 18 \mathrm{~nm}$ TMV vertical viruses in $0.1 \mathrm{M}$ phosphate buffer ( $\mathrm{pH} 7$ ) for 18 hours, allowing for self-assembly of the virus particles on the microfabricated planar gold electrodes via the previously mentioned cysteine residues. Following TMV selfassembly, nickel was electroless deposited for 5 minutes [19]. Sequentially, gold was electroless plated in a gold plating bath $\left(0.007 \mathrm{M} \mathrm{KAu}(\mathrm{CN})_{2}, 1.4 \mathrm{M} \mathrm{NH} \mathrm{Nl}_{4}, 0.2 \mathrm{M}\right.$ Sodium citrate, and $1 \mathrm{M}$ $\mathrm{NaH}_{2} \mathrm{PO}_{2}$. $\mathrm{pH}$ 7.0-7.5) for 45 minutes at $90^{\circ} \mathrm{C}$ to form a conformal gold coating on the virus particles. Fig. 1 shows SEM images of the surface of the electrode modified with nickel-coated TMV-1cys after gold deposition (TMV/Ni/Au modified electrode). TMV- 1cys were observed to attach vertically on the electrode surface. A TEM image of the nanocomposite virus particle (Fig. 2) shows approximately $67 \mathrm{~nm}$ nickel and $46 \mathrm{~nm}$ gold coatings. The thin films of the nickel and the gold maintain the high aspect ratio of the TMV scaffold after plating, increasing the electrochemically active surface area of the electrode.
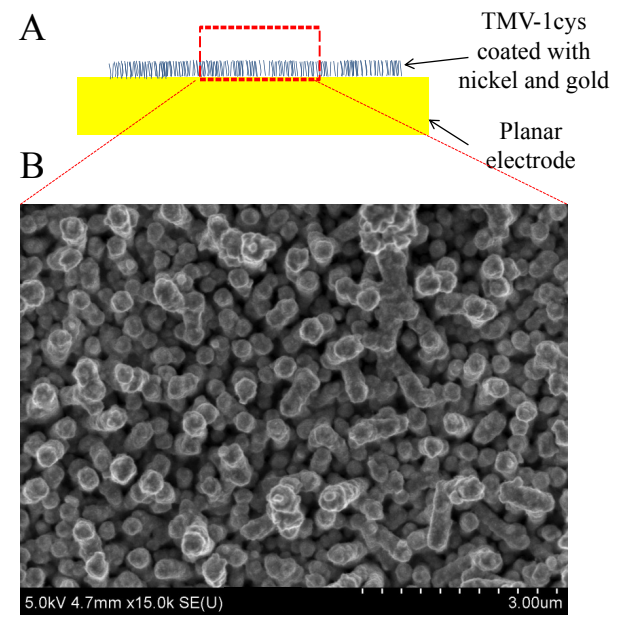

Figure 1: Self-assembled TMV-1cys molecules coated with nickel and gold layers on the surface of planar electrode. (A) Cross section schematic. (B) Scanning electron micrograph.

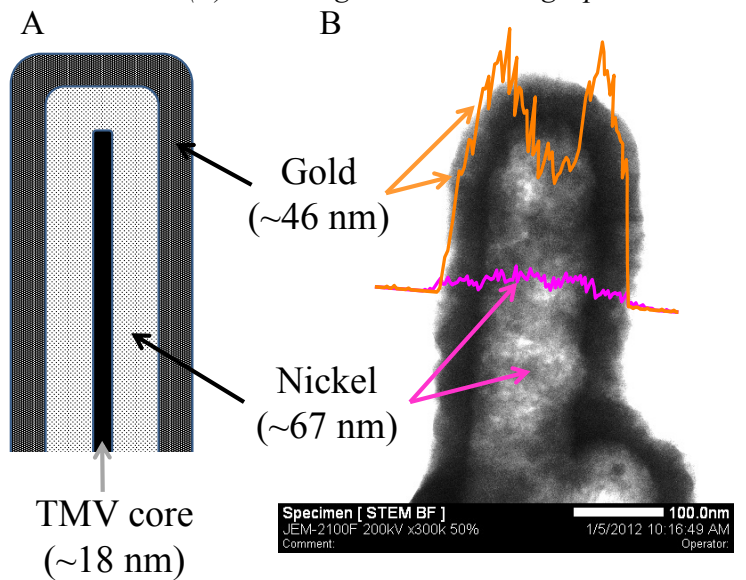

Figure 2: (A) Schematic of the TMV coatings. (B) Transmission electron microscopy analysis of the TMV-1cys coated with nickel and gold layers with EDS profiles of nickel (pink / dark gray) and gold (orange / light gray).

The modified electrodes were electrochemically characterized using cyclic voltammetry (CHI660D single channel potentiostat from $\mathrm{CH}$ Instruments, Austin, TX. Commercial Ag/AgCl reference electrode. Pt wire counter electrode) in the presence of a redox couple $5 \mathrm{mM}$ ferrocyanide, $5 \mathrm{mM}$ ferricyanide, $10 \mathrm{mM}$ phosphate buffers saline (PBS) solution. Fig. 3 illustrates the increased signal from the modified electrodes compared to unmodified electrode with cyclic voltammograms of the generated electrochemical reduction and oxidation reactions. The electrodes produced reversible nernstian characteristics where the $\mathrm{TMV} / \mathrm{Ni} / \mathrm{Au}$ modified electrode generated the highest electrochemical current (8-fold higher oxidation peak current in comparison with the unmodified planar electrode) due to the high active surface area. The oxidation and the reduction peaks generated by the $\mathrm{TMV} / \mathrm{Ni} / \mathrm{Au}$ modified electrodes varied in compare to the other types of electrodes. This variation is may be due to increase with the uncompensated resistance in the new high aspect ratio electrochemical interface. Furthermore, the potential presence of 
nickel uncoated with gold on the TMV scaffold may result in changes with the standard reduction potential of the redox couple. The increased electrochemical activity of the $\mathrm{Ni} / \mathrm{Au}$ modified electrode compared to unmodified electrode may be due high surface roughness resulted by the nickel and the gold electroless plating. Furthermore, the TMV modified electrode impeded the generated electrochemical current that was attributed to the nonconductive virus coating on the electrode surface.

The effective surface area of the unmodified and the modified electrodes were calculated from Bard and Faulkner [27]:

$$
I_{\text {peak }}=0.4463\left(F^{3} / R T\right) n^{3 / 2} A D^{1 / 2} C^{*} v^{I / 2}
$$

Where $F\left[\mathrm{C} \mathrm{mol}^{-1}\right]$ is the Faraday constant, $R\left[\mathrm{~J} \mathrm{~mol}^{-1} \mathrm{~K}^{-1}\right]$ is the gas constant, $T[\mathrm{~K}]$ is the temperature, $n$ is the stoichiometric number of electrons involved in an electrode reaction, $A\left[\mathrm{~cm}^{2}\right]$ is the effective surface area of the electrode, $D\left[\mathrm{~cm}^{2} \mathrm{~s}^{-1}\right]$ is the diffusion coefficient of the electro-active species, $C^{*}\left[\mathrm{~mol} \mathrm{~cm}^{-3}\right]$ is the bulk concentration of the electro-active species, $v\left[\mathrm{~V} \mathrm{~s}^{-1}\right]$ is the linear potential scan rate, and $I_{\text {peak }}[\mathrm{A}]$ is the peak current. Effective surface area calculations demonstrated that TMV/Ni/Au resulted in the highest area (Table 1), a characteristic important for the high performance of electrochemical sensors due to the surface area limited reaction kinetics.

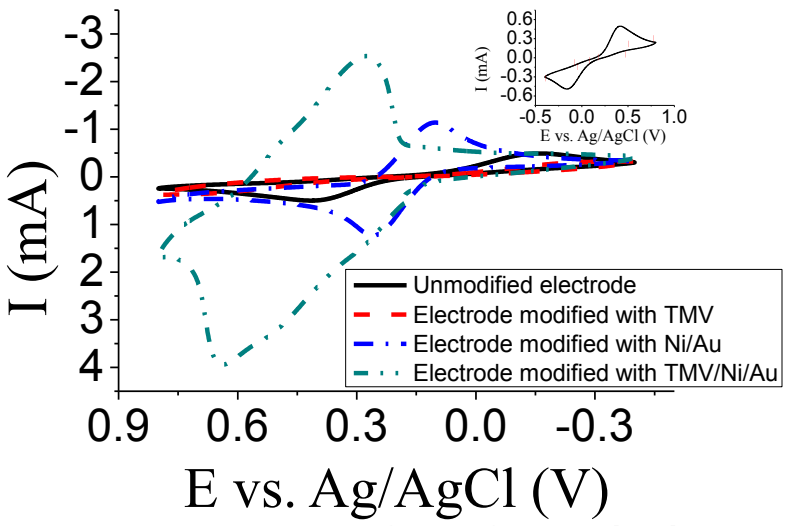

Figure 3: Cyclic voltammograms of the planar electrode modified with either the TMV, the Ni/Au electroless deposition, or both. Inset shows only the unmodified and the TMV modified electrodes.

Table 1: Measured effective surface area and bio-detection efficiency analysis for the unmodified and the modified electrodes.

\begin{tabular}{|c|c|c|}
\hline $\begin{array}{c}\text { Electrode } \\
\text { modification }\end{array}$ & $\begin{array}{c}\text { Effective } \\
\text { surface area } \\
{\left[\mathbf{c m}^{2}\right]}\end{array}$ & $\begin{array}{c}\text { Bio-detection } \\
\text { efficiency [\%] }\end{array}$ \\
\hline Unmodified & 0.42 & 1.7 \\
\hline $\mathrm{TMV}$ & 0.32 & 2.1 \\
\hline $\mathrm{Ni} / \mathrm{Au}$ & 1.05 & $\begin{array}{c}-1.1 \text { (poor } \\
\text { efficiency) }\end{array}$ \\
\hline $\mathrm{TMV} / \mathrm{Ni} / \mathrm{Au}$ & 3.34 & $\mathbf{1 6 . 1}$ \\
\hline
\end{tabular}

In this study the target NRG1 polymorphism ssDNA (SNP8NRG243177) bio-detection efficiency was electrochemically analyzed using thiolated ssDNA probe to NRG1 assembled on the high surface area $\mathrm{TMV} / \mathrm{Ni} / \mathrm{Au}$ modified electrode. In short, electrodes were incubated in a solution containing $10 \mathrm{mM}$ PBS, $100 \mathrm{mM} \mathrm{NaCl}, 10 \mu \mathrm{M}$ Tris (2carboxyethyl) phosphine (TCEP) and $1 \mu \mathrm{M}$ probe ssDNA for 3 hour followed by rinsing with PBS. Afterwards, the electrodes were incubated for 24 hour in PBS solution containing $1 \mathrm{mM}$ of 6mercapto-1-hexanol (MCH). $\mathrm{MCH}$ is used to passivate any exposed regions on the surface to reduce non-specific binding during DNA hybridization. Incubation with either the noncomplementary or the target sequences was performed in a $4 x$ saline sodium citrate (SSC) buffer containing $1 \mu \mathrm{M}$ of the target DNA for 1 hour.

Biosensing performance was studied with cyclic voltammetry in the presence of the previously described redox ferrocyanide/ferricyanide solution (Fig. 4). All electrodes demonstrated reversible nernstian characteristics in the presence of complementary NRG1 target and non-complementary ssDNA. Moreover, the TMV/Ni/Au modified electrode demonstrated the largest current differentiation upon DNA hybridization. DNA hybridization causes stronger electrostatic repulsion forces with the negatively charged electro-active species in the electrolyte, hence impeding the electrochemical reaction at the electrode [28]. Biodetection efficiency (ratio of oxidation peak current decrease between non-complementary and complementary ssDNA) analysis (Table 1) demonstrated 9.5-fold improved bio-detection performance for the 3D TMV/Ni/Au modified electrode compared to the unmodified electrode. This increase is not only due to the higher effective surface area (factor of 8), but also due to the inherent properties of nanomaterials improving biosensing performance, e.g. augmented amount of probes functionalizing the surface of the electrode and heightened target concentration near the electrochemically reactive surface.

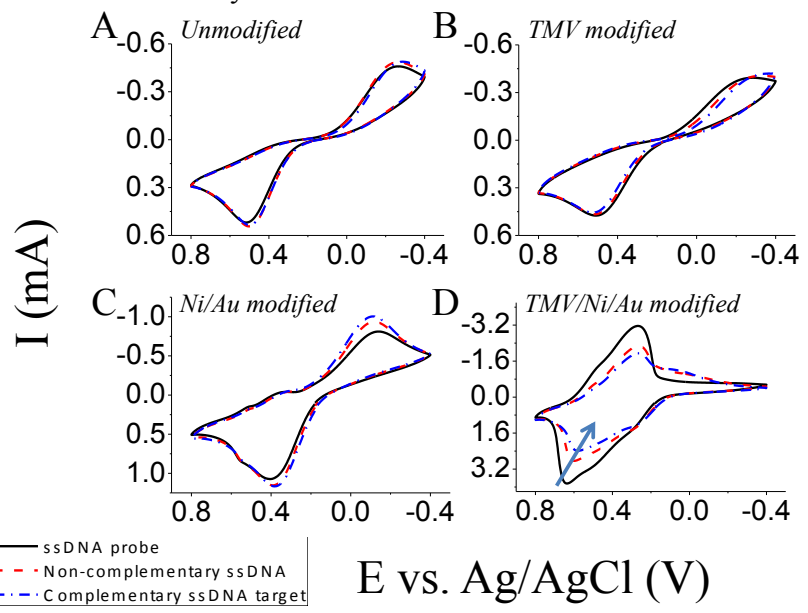

Figure 4: Cyclic voltammograms of the (A) planar unmodified electrode, (B) TMV modified electrode, (C) Ni/Au modified electrode, and (D) TMV/Ni/Au modified electrode following introduction of ssDNA NRG1 probe (black solid), noncomplementary sSDNA (red dashed), and complementary NRG1 target SSDNA (blue dashed-dot). Arrow indicates current reduction upon DNA hybridization.

\section{CONCLUSIONS}

This work demonstrated the first utilization of TMV molecules coated with nickel and gold as nano-scale biotemplates assembled on an electrochemical biosensor for schizophrenia analysis. The biotemplated TMV modified sensor generated heightened electrochemical activity that improved the biosensing performance. Given the large set of biological recognition events that can be detected electrochemically and the innately high compatibility of TMV-biotemplate integration with conventional microfabrication technology, we believe this work has broad applications in biological electrochemical sensing and transduction. Specifically, we envision the utilization of TMV as a high aspect ratio nano-scale 3D scaffold for high surface area microelectrodes integrated in MEMS sensing devices improving 
dramatically their performance. We believe TMV-biotemplated LOC devices leverage electrochemical biosensing reactions that will lead to the creation of new families of low-cost, rapid and sensitive miniaturized biosensors for point-of-care analysis.

\section{ACKNOWLEDGMENTS}

The authors acknowledge the Robert W. Deutsch Foundation and National Science Foundation Emerging Frontiers in Research and Innovation (EFRI) for financial support. The authors also thank the Maryland Nanocenter and its Fablab for cleanroom facility support. The authors grateful to Prof. Yosi ShachamDiamand and Prof. Alexandra Inberg from the Tel Aviv University for the fruitful discussion on gold electroless deposition.

\section{REFERENCES}

[1] A. Le Goff, M. Holzinger, and S. Cosnier, "Enzymatic Biosensors based on SWCNT-Conducting Polymer Electrodes", Analyst, 136, 1279 (2011).

[2] W. Siangproh, W. Dungchai, P. Rattanarat, and O. Chailapakul, "Nanoparticle-based Electrochemical Detection in Conventional and Miniaturized Systems and Their Bioanalytical Applications: A Review", Analytica Chimica Acta, 690, 10, (2011).

[3] R.H. Baughman, A.A. Zakhidov, and W.A. de Heer, "Carbon Nanotubes - The Route Toward Applications”, Science, 297, 787 (2002).

[4] J. Wang, "Carbon-Nanotube based Electrochemical Biosensors: A Review”, Electroanalysis, 17, 7 (2005).

[5] J.-S. Ye, Y. Wen, W. De Zhang, L. Ming Gan, G.Q. Xu, and F.-S. Sheu, "Nonenzymatic Glucose Detection using Multiwalled Carbon Nanotube Electrodes", Electrochemistry Communications, 6, 66 (2004).

[6] D.J. Evans, "The Bionanoscience of Plant Viruses: Templates and Synthons for New Materials", Journal of Materials Chemistry, 18, 3746 (2008).

[7] J.C. Smith, K.-B. Lee, Q. Wang, M.G. Finn, J.E. Johnson, M. Mrksich, and C.A. Mirkin, "Nanopatterning the Chemospecific Immobilization of Cowpea Mosaic Virus Capsid", Nano Letters, 3, 883 (2003).

[8] K. Namba, R. Pattanayek, and G. Stubbs, "Visualization of Protein-Nucleic Acid Interactions in a Virus: Refined Structure of Intact Tobacco Mosaic Virus at $2.9 \AA$ Resolution by X-ray Fiber Diffraction", Journal of Molecular Biology, 208, 307 (1989).

[9] A.C. Durham, J.T. Finch, and A. Klug, "States of Aggregation of Tobacco Mosaic Virus Protein", Nature New Biology, 229, 37 (1971).

[10] J.N. Culver, W.O. Dawson, K. Plonk, and G. Stubbs, "SiteDirected Mutagenesis Confirms the Involvement of Carboxylate Groups in the Disassembly of Tobacco Mosaic Virus", Virology, 206, 724 (1995).

[11] J.N. Culver, "Tobacco Mosaic Virus Assembly and Disassembly: Determinants in Pathogenicity and Resistance", Annual Review of Phytopathology, 40, 287 (2002).

[12] M. Knez, M. Sumser, A.M. Bittner, C. Wege, H. Jeske, T.P. Martin, and K. Kern, "Spatially Selective Nucleation of Metal Clusters on the Tobacco Mosaic Virus", Advanced Functional Materials, 14, 116 (2004).

[13] M. Knez, A. Kadri, C. Wege, U. Gösele, H. Jeske, and K. Nielsch, "Atomic Layer Deposition on Biological Macromolecules: Metal Oxide Coating of Tobacco Mosaic Virus and Ferritin", Nano Letters, 6, 1172 (2006).

[14] M. Knez, M. Sumser, A.M. Bittner, C. Wege, H. Jeske, S.
Kooi, M. Burghard, and K. Kern, "Electrochemical Modification of Individual Nano-Objects", Journal of Electroanalytical Chemistry, 522, 70 (2002).

[15] R.J. Tseng, C. Tsai, L. Ma, J. Ouyang, C.S. Ozkan, and Y. Yang, "Digital Memory Device based on Tobacco Mosaic Virus Conjugated with Nanoparticles", Nature Nanotechnology, 1, 72 (2006).

[16] S.-Y. Lee, E. Royston, J.N. Culver, and M.T. Harris, "Improved Metal Cluster Deposition on a Genetically Engineered Tobacco Mosaic Virus Template", Nanotechnology, 16, S435 (2005).

[17] H. Yi, S. Nisar, S.-Y. Lee, M.A. Powers, W.E. Bentley, G.F. Payne, R. Ghodssi, G.W. Rubloff, M.T. Harris, and J.N. Culver, "Patterned Assembly of Genetically Modified Viral Nanotemplates via Nucleic Acid Hybridization", Nano Letters, 5, 1931 (2005).

[18] K. Gerasopoulos, M. McCarthy, P. Banerjee, X. Fan, J.N. Culver, and R. Ghodssi, "Biofabrication Methods for the Patterned Assembly and Synthesis of Viral Nanotemplates", Nanotechnology, 21, 055304 (2010).

[19] K. Gerasopoulos, M. McCarthy, E. Royston, J.N. Culver, and R. Ghodssi, "Nanostructured Nickel Electrodes using the Tobacco mosaic virus for Microbattery Applications", Journal of Micromechanics and Microengineering, 18, 104003 (2008).

[20] E. Pomerantseva, K. Gerasopoulos, X. Chen, G. Rubloff, and R. Ghodssi, "Electrochemical Performance of the Nanostructured Biotemplated V2O5 Cathode for Lithium-ion Batteries”, Journal of Power Sources, 206, 282 (2012).

[21] K. Gerasopoulos, X. Chen, J. Culver, C. Wang, and R. Ghodssi, "Self-assembled Ni/TiO2 Nanocomposite Anodes Synthesized via Electroless Plating and Atomic Layer Deposition on Biological Scaffolds", Chemical Communications, 46, 7349 (2010).

[22] R.A. Carlstedt, Handbook of Integrative Clinical Psychology, Psychiatry, and Behavioral Medicine: Perspectives, Practices, and Research, Springer Publishing Company, New York, 2010.

[23] B.J. Sadock, H.I. Kaplan, and V.A. Sadock, Kaplan \& Sadock's Synopsis of Psychiatry: Behavioral Sciences/Clinical Psychiatry, $10^{\text {th }}$ ed., Lippincott Williams \& Wilkins, Philadelphia, 2007.

[24] B. Rico and O. Marín, "Neuregulin Signaling, Cortical Circuitry Development and Schizophrenia", Current Opinion in Genetics \& Development, 21, 262 (2011).

[25] P.J. Harrison and A.J. Law, "Neuregulin 1 and Schizophrenia: Genetics, Gene Expression, and Neurobiology", Biological Psychiatry, 60, 132 (2006).

[26] T. Walsh, J.M. McClellan, S.E McCarthy, A.M. Addington, S.B. Pierce, G.M. Cooper, A.S. Nord, M. Kusenda, D. Malhotra, A. Bhandari et al., "Rare Structural Variants Disrupt Multiple Genes in Neurodevelopmental Pathways in Schizophrenia", Science, 320, 539 (2008).

[27] A.J. Bard and L.R. Faulkner, Electrochemical Methods: Fundamentals and Applications, John Wiley \& Sons, New York, 2001.

[28] E. Katz and I. Willner, "Probing Biomolecular Interactions at Conductive and Semiconductive Surfaces by Impedance Spectroscopy: Routes to Impedimetric Immunosensors, DNASensors, and Enzyme Biosensors", Electroanalysis, 15, 913 (2003).

\section{CONTACT}

*Reza Ghodssi, tel: +1-301-405-8158; GHODSSI@UMD.edu Hadar Ben-Yoav, tel: +1-301-405-2168; BENYOAV@,UMD.edu 